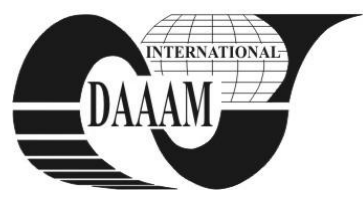

Annals of DAAAM for 2011 \& Proceedings of the 22nd International DAAAM Symposium, Volume 22, No. 1, ISSN 1726-9679 ISBN 978-3-901509-83-4, Editor B. Katalinic, Published by DAAAM International, Vienna, Austria, EU, 2011 Make Harmony between Technology and Nature, and Your Mind will Fly Free as a Bird Annals \& Proceedings of DAAAM International 2011

\title{
FRONTAL HARMONIC TRANSMISSIONS (FHT): STUDY OF ELASTIC DEFORMATIONS OF THE FLEXIBLE WHEEL
}

\author{
KAPOSTA, I[osif] \& OTLACAN, D[imitrie] D[anut]
}

\begin{abstract}
The aim of the paper is to succinctly present a new family of harmonic cogged transmission, namely, the frontal ones - construction, functioning, and advantages. From the facts submitted it results unambiguously that establishing the profile of the teeth of the flexible wheel with frontal teething depends on the distortion law imposed by the wave generator, a law which determines the spatial state of distortions and tensions of the body of this wheel.
\end{abstract}

Key words: harmonical drive, mechanism, gear, flexible wheel, distortion

\section{INTRODUCTION}

Given the multiple advantages demonstrated by harmonic transmissions, they have drawn considerable interest ever since their launch.

In order to eliminate the main shortcomings of radial harmonic transmissions currently being manufactured shortcomings mainly due to the constructive shape of the flexible cogwheel and the type of alternating-symmetrical bending stress occurring in the body of the flexible wheel and of the special ball bearing - there have been new variants developed, that can diminish and even eliminate these disadvantages.

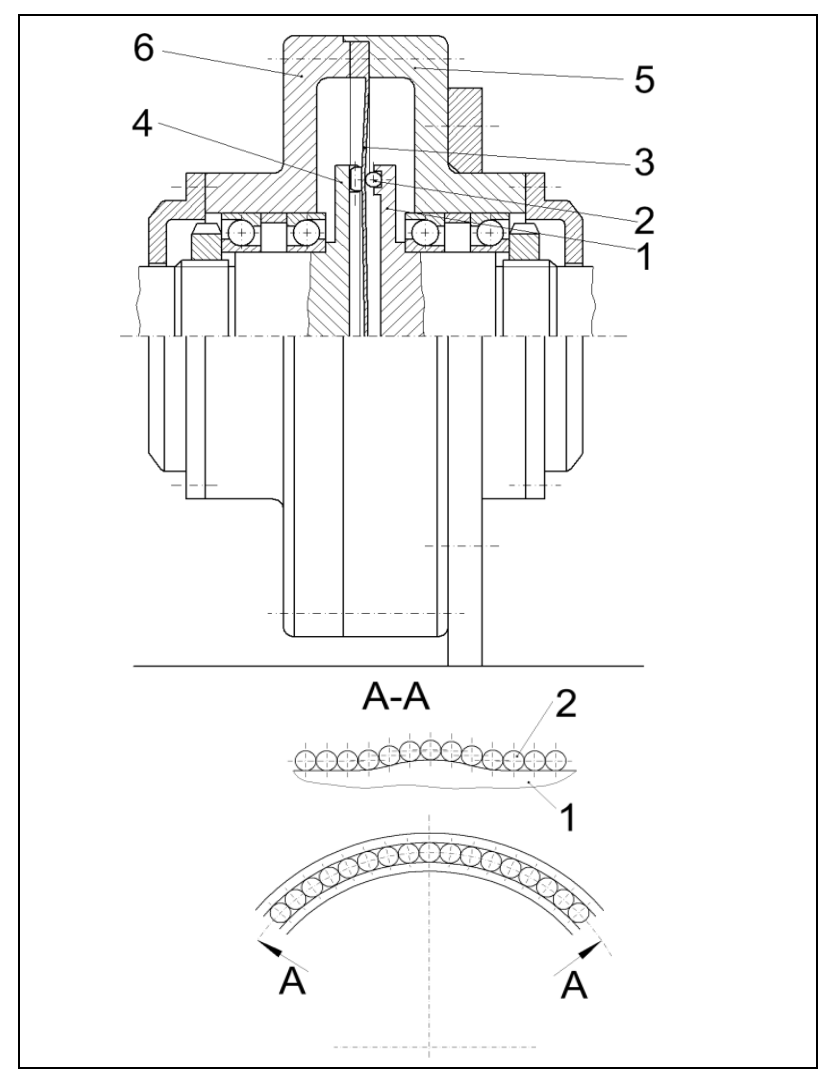

Fig.1. Frontal Harmonical transmnission - general view
In this context, the frontal harmonic transmission (FHT) ( Kaposta, 19848) constitutes an innovative solution that eliminates the two major disadvantages mentioned above, while significantly diminishing the axial gauge as well, given that the flexible cogwheel is shaped as a circular plate with frontal teeth.

Thus, the harmonic frontal transmission provides several additional advantages versus radial harmonic transmissions, given equivalent kinematic and kinetostatic conditions:

- constructive and technological simplicity of the flexible cogwheel (which can be executed through one single precision press forging operation with very high deformation speed);

- fiber disposition continuity, fatigue resistance of the flexible wheel with frontal teeth is $\sim 70 \%$ higher, and is further advantaged by a pulsating stress cycle as opposed to an alternating-symmetrical cycle;

Drawing 1 illustrates a conceptual constructive solution of this transmission, where: 1 - waves generator, 2 - distortion balls, 3 - flexible wheel with frontal teeth and a number $z_{f}$ of teeth, 4 - rigid wheel with frontal teeth and a number $z_{r}$ of teeth, 5 - anterior semi-housing, 6 - posterior semi-housing.

How does the transmission work: following the mounting of the waves generator (using a frontal cam), it will deform the flexible cogwheel in its axial direction using the balls (2), that are rolling though a circular canal of variable depth found on the frontal surface of the waves generator. Through axial deformation, the teeth of the flexible cogwheel will gear up with the teeth of the rigid wheel, in the area of the axial deformation.

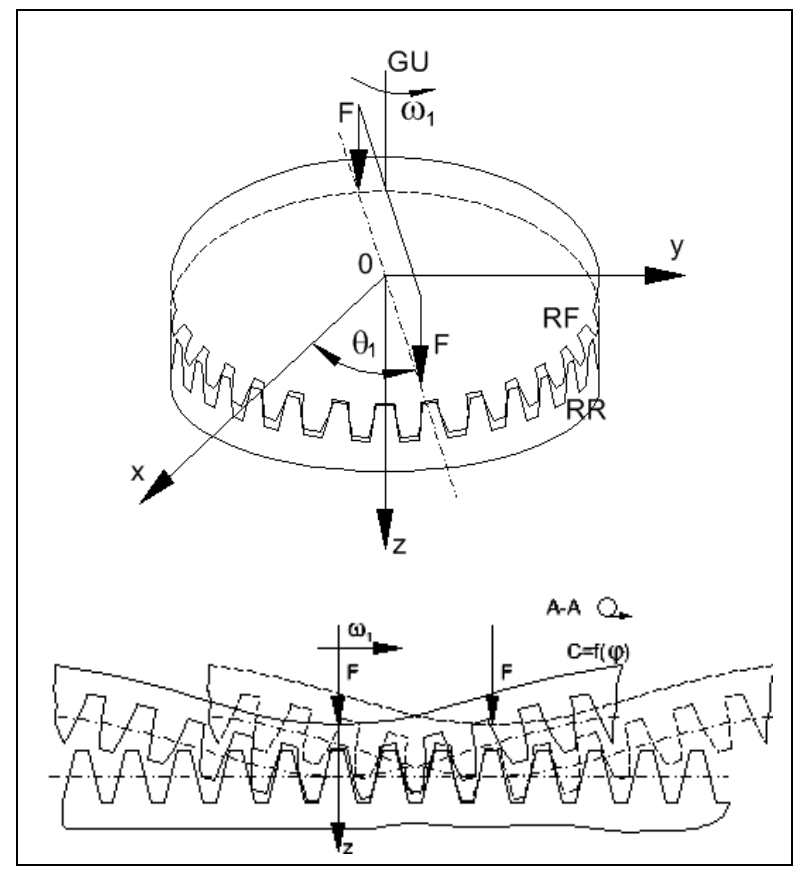

Fig.2. Frontal Harmonical transmnission - forced gearing 
The rotation of the rigid wheel occurs due to the fact that the generator is set into a rotating movement imposed by the difference in the number of teeth of the two wheels that are in forced gear.

We consider that, at a first approximation, the flexible cogwheel with frontal teeth can be assimilated to a thin circular plate with small distortions, being put under stress by asymmetrical concentrated forces that act in the direction of the normal on the distorted surface, as per Drawing 2.

The correct choice of the law of distortion of the flexible wheel - law which is imposed on the latter by the wave generator - has a determining influence upon the energetic, kinematic performances and upon the durability of the transmission.

At the same time, the distortion law imposed by the wave generator has to the same with native distorting law of the circular plate that put under stress by concentrated, identical and equidistant forces corresponding to the distorting forces of the wave generator. In the opposite case, additional stresses can be foreseen, that are difficult to evaluate and have negative effects upon the durability and the efficiency of the transmission.

\section{DIFERENTIAL EQUATION OF THE DISTORTED SURFACE OF THE FLEXIBLE WHEEL}

In order to carry out the theoretic study of the plane state of tensions and distortions of the flexible cogwheel with frontal teeth, the following hypotheses have been adopted:

- the width of the flexible cogwheel remains constant on the whole surface, neglecting local stiffening incurred by the teeth;

- the distortion of the flexible cogwheel takes place under the action of the wave generator, whose distorting force is equivalent to a concentrated force applied to the middle of the teeth.

Under these conditions, the flexible cogwheel was assimilated with a circular thin plate with small distortions, embedded on the exterior outline, and put under stress by a concentrated force applied to the middle of the teeth in the direction normal on the distorted surface.

The differential equation of the distorted surface is given by the relation (Timosenko, 1968) below:

$$
\mathrm{w}=\left[\left(\frac{\partial^{2}}{\partial \mathrm{r}^{2}}+\frac{1}{\mathrm{r}} \cdot \frac{\partial}{\partial \mathrm{r}}+\frac{1}{\mathrm{r}^{2}} \cdot \frac{\partial^{2}}{\partial \theta^{2}}\right)\left(\frac{\partial^{2} \mathrm{w}}{\partial \mathrm{r}^{2}}+\frac{1}{\mathrm{r}} \cdot \frac{\partial \mathrm{w}}{\partial \mathrm{r}}+\frac{1}{\mathrm{r}^{2}} \cdot \frac{\partial^{2} \mathrm{w}}{\partial \theta^{2}}\right)\right]=\frac{\mathrm{q}}{\mathrm{D}}
$$

where: $\mathrm{w}[\mathrm{mm}]-$ is the travel in transversal direction; $\mathrm{q}$ $\left[\mathrm{N} . \mathrm{mm}^{2}\right]$ - is the transversal load distributed onto the surface of the plate; $\mathrm{r}[\mathrm{mm}]-$ momentary radius; $\mathrm{D}[\mathrm{mNm}]-$ is the rigidity modulus at cylindrical bending, given by the equation:

$$
\mathrm{D}=\frac{\mathrm{E} \cdot \mathrm{h}^{3}}{12 \cdot\left(1-\mathrm{v}^{2}\right)} \quad[\mathrm{mNm}]
$$

In equation (2) we have noted with $\mathrm{E}$ [Mpa] the elasticity modulus of the material of the flexible wheel; $\mathrm{v}$ [ - ] - the coefficient of Poisson; $\mathrm{h}[\mathrm{mm}]$ the width of the flexible wheel.

Two methods have been employed in order to solve the equation (1): Clebsh (1852) (Timosenko 1968) and Mitchell (L'Hermite 1953).

\section{CONCLUSIONS}

There are significant differences between the two methods, with regard to the values of deformations and of the tensions.

These differences are caused by the simplifying hypotheses employed by Clebsch and Mitchell; as well as the impossibility to describe with high accuracy the exact fixation of the flexible cogwheel and the actual interaction between the waves generator and the deformed area of the flexible cogwheel, respectively.

Since these issues are of great importance for the correct functioning of the frontal harmonic transmission, we have conducted an experimental investigation of the real state of deformation, using actual flexible cogwheel solutions. The results of this experimental investigations are subsequently compared with the theoretical results, as shown in Drawing 3 and Drawing 4. We can therefore conclude that the Mitchell method offers results that are very close to those experimentally determined.

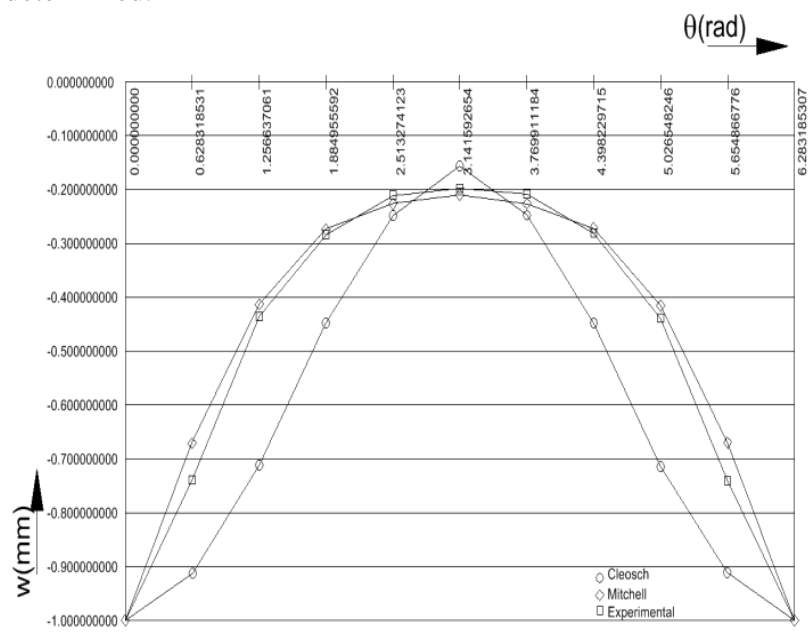

Fig.3. Deformations along the circumference

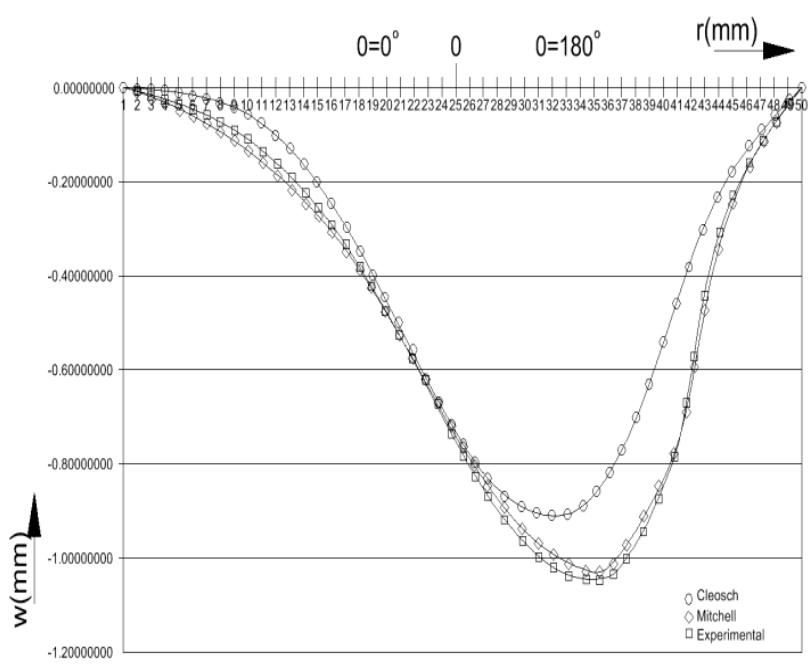

Fig. 4. Deformations on diametrical direction

\section{REFERENCES}

L'Hermite, (1953). Rézistance des matériaux,L'Inginerie Nouvelle, Orleans

Kaposta, I. (1984). Frontal harmonical transmission (in Romanian), Patent Romania, No. 89975

Kaposta, I. (1995). Optimizing the functional design of the frontal harmonical transmission (in Romanian), Doctoral Thesis, University "Politehnica" Timisoara,

Kaposta, I. (1998). Contribution to the mathematical modeling of functional processes, Buletins for Aplied\&Computer Mathematics-1493/1998-LXXXV-B, Technical University of Budapest

Timosenko, S. (1968). Theory of flat and curved plates (in Romanian), Technical Publishing, Bucharest 